\title{
Anesthesia without opioids
}

\author{
Mohsin Nazir Butt ${ }^{1}$, Asma Faraz ${ }^{2}$, Maheen Fazal ${ }^{2}$ \\ 1. Assistant Professor, Department of Anesthesiology, Aga Khan University, Karachi, Pakistan. \\ 2. Research Associate, Department of Anesthesiology, Aga Khan University, Karachi, Pakistan.
}

Correspondence: Dr. Mohsin Nazir Butt; E-mail: mohsin.nazir@aku.edu; Phone: +92 3212227083

\section{Summary}

Opium was the first opioids with sedative and analgesic effects, so was readily adopted by the surgical doctors to be used during surgeries. Later on its natural alkaloid, morphine, was extensively used, till synthetic alkaloids were introduced in the clinical practice. Now many non-narcotic analgesic drugs have been synthesized and proven to be devoid of serious side effects, commonly associated with the opioid use. There have been some staunch advocates of non-narcotic based anesthesia, who claim that the side effects of the opioids outweigh their benefits in the anesthesia. This editorial throws some light on the current and the future trends in analgesic use in anesthesia.

Key words: Opioids; Analgesics; Non-opioid analgesics; Perioperative pain; Side effects

Citation: Butt MN, Faraz A, Fazal M. Anesthesia without opioids (Editorial). Anaesth. pain intensive care 2021;26(1):4-7; DOI: 10.35975/apic.v26i1.1758

Received: January 10, 2022, Accepted: January 12, 2022

\section{Opioids}

Opioids are considered as an integral part of perioperative management because of their relatively high analgesic efficacy as compared to the non-opioid analgesics. Natural opiates like opium, have been used for thousands of years but the practise of using synthetic opioids started after the second world war i.e.,1950s. ${ }^{1}$ In 1962, fentanyl was used for anaesthesia as the first synthetic opioid. It was believed as a milestone in the field of anaesthesia and a true revolution in anaesthesia practise. Due to the remarkable intraoperative haemodynamic stability, fentanyl was added in the anaesthesia protocol as a component of balanced anaesthesia. $^{1}$

The concept of balanced general anaesthesia includes reversible unconsciousness, amnesia, immobility and antinociception. $^{2}$ It has been described that the nociceptive pathway has significant connections with arousal pathway. Under general anaesthesia, opioids are the primary agents of anti-nociception, decreasing arousal by acting on different receptors. ${ }^{2}$ Opioids, as an adjuvant in general anaesthesia have been observed to decrease the need of hypnotics during induction and maintenance phases of anaesthesia and also blunt the laryngoscopy and intubation response during airway management. ${ }^{3}$ The utilization of opioids during intraoperative stages of surgical procedures and postoperatively has also been approved by Food and Drug Administration (FDA) authority. ${ }^{4}$ In the 1990s, the use of opioid analgesics was highly recommended to deal with chronic pain in both developed and developing countries. ${ }^{5}$ Even now, it is prescribed for relieving pain in almost all patient care settings. However, the preference and dose of opioid analgesics varies between the management of perioperative pain and chronic pain.

The commonly observed adverse effects of opioids include nausea, vomiting, dizziness, shivering, impaired gastrointestinal function, urinary retention, and pruritus in acute pain settings. ${ }^{6,7}$ Further consumption of large doses of opioids can result in acute tolerance and hyperalgesia. ${ }^{8}$ Opioid induced hyperalgesia increases the sensitivity to pain which can be resolved after discontinuation of opioids. Other efficacious adverse effects include sedation and respiratory depression. ${ }^{9}$ Respiratory problems are also the result of high dose, misuse, or overdose. Therefore, it is essential to control or balance the usage of opioids, multimodal analgesia and opioid-sparing in relieving pain. ${ }^{10}$ The use of opioid analgesics is a risk in public health and has been associated with undesirable events like abuse, addiction, misuse, and overdose leading to death when used for chronic non-cancer pain. ${ }^{11}$ 


\section{Global opioid crisis}

The current global opioid crisis has multifactorial reasons worsened gradually due to the different waves of liberal opioid use. Starting from the first wave in the 1960s with excessive use of opioids for acute pain management, second wave in the 1990s with use of oral opioid for chronic pain despite vague evidence in the medical literature and the third wave from 2001-2010 with expended use of oral opioids due to inappropriate marketing and liberalised prescribing. ${ }^{12}$ The ongoing opioid crisis has led to the development of alternative techniques like opioid sparing and opioid free anaesthesia strategies.

\subsection{Opioid sparing and opioid free anesthesia (OFA)}

Despite the well-known analgesic efficacy of opioids, use of multimodal analgesia and opioid sparing have their role in reducing the opioid-related adverse effects. Owing to the well-researched and recognized opioidbased anaesthesia adverse effects, it was essential to develop alternative therapies to achieve the main benefits of opioids like analgesia and haemodynamic stability, while limiting the opioid related adverse effects. The multimodal regimen consists of non-opioid pharmacologic techniques and regional anaesthetic techniques (peripheral and central neuraxial). The diverse mechanisms of the pharmacological agents provide the advantage of multiple modes of action to achieve intraoperative and postoperative analgesia. The pharmacological agents used are NMDA antagonists, Ketamine and Lidocaine; anti- Inflammatory drugs, NSAIDs and Dexamethasone; Alpha-2-Agonists, Dexmedetomidine, Clonidine, and Tizanidine; Anticonvulsant, Gabapentin, and Pregabalin. The regional anaesthesia or neuraxial blocks work by reducing the stress response and blood loss, thereby reducing the requirement for transfusion. ${ }^{13}$ However, the amount of intraoperative opioid sparing differs among patients due to the variation of surgical procedures and analgesic requirements.

The Concept of Opioid Free Anaesthesia (OFA) is not new. The term "balanced anaesthesia" was introduced in 1962 and earned recognition later. However, it did not particularly focus on opioid presence in it. Later in 1989, the concept opioid and non-opioid combinations was developed in anaesthesia practice. ${ }^{14}$ A study conducted in 2012 by Mulier proposed OFA for obese patients. ${ }^{15}$ Opioid free anaesthesia (OFA) can be defined as the combination of various opioid-sparing techniques leading to disappearance of intraoperative opioids. ${ }^{1}$ These techniques are applied to avoid short and longterm side effects of opioids. At the same time, they are providing more effective pain control and reducing the reliance on opioids. Thus, ensuring adequate analgesia control and optimizing postoperative recovery. ${ }^{16}$ Many physicians and medical providers aim to provide OFA by eliminating the intake of opioids intraoperatively. ${ }^{17}$ Opioid sparing is an appropriate technique to avoid excessive opioid administration and their subsequent adverse effects, while complete opioid elimination may not be as effective as it was thought. In a recent review article, it has been concluded that the opioid-free strategies are not superior to the opioid-sparing techniques. $^{3}$

\section{Discussion}

The opioid-sparing approach has been suggested to be the best intervention to prevent the development of opioid-associated delirium. ${ }^{18}$ A lower incidence of postoperative ileus and a shorter duration of hospital stay have also been observed with the use of opioids sparing liposomal bupivacaine. ${ }^{19}$ When it comes to postoperative pain outcomes, opioid-inclusive anaesthesia does not show much of an advantage over opioid-free techniques. It was observed by Frauenknecht et al. that the opioidfree regimen also resulted in a $20 \%$ decrease in postoperative nausea and vomiting. ${ }^{20}$ Mohamed et al. reported that general anaesthesia without opioids is equally effective in bariatric surgery. ${ }^{21}$ Hence, there is plenty of evidence that the multimodal, opioid-sparing approach can be deemed an effective strategy for effective pain management, improved quality of care, and most importantly, reduced consumption of opioids postoperatively.

The Multimodal approach constitutes a combination of drugs from different classes, which means that it is essential to be aware of drug interactions. In a recent randomised controlled trial by Beloeil $\mathrm{H}$. et al., balanced opioid-free anaesthesia with dexmedetomidine was compared with balanced opioid anaesthesia with remifentanil. It concluded that, compared to the balanced anaesthesia with remifentanil, the opioid-free anaesthesia with dexmedetomidine had more serious adverse effects, i.e., severe bradycardia, hypoxaemia, longer time to extubation, and longer post anaesthesia care unit stay. ${ }^{22}$ Certain Adjuncts such as lidocaine and dexmedetomidine also have known vasoactive effects resulting from their toxically high levels in plasma in some cases. ${ }^{23,24}$

The use of opioid-sparing and opioid-free anaesthesia techniques is preferable in developing countries like Pakistan. Generally, developing countries' healthcare systems are deficient in funding, infrastructure, facilities, qualified healthcare personnel, and advanced equipment. These are thought to be due to inefficient healthcare planning and a low healthcare budget. In addition, most of the pharmaceutical products, their precursors, and raw material are being imported from other countries. Opioids for medical use are controlled drugs, and 
usually, most of the opioids are imported from different countries of the world. Drug Regulatory Authority of Pakistan (DRAP) regulates the distribution of opioids and other controlled drugs within the country. It has a well-defined quota of opioids for each region of the country and each healthcare institution. In the last one to two decades, persistent increases in opioid requirement in chronic pain and relatively low supply have led to the deficiency of opioids for anaesthesia use. The gradual shift from balanced opioid anaesthesia to opioidsparing/opioid-free anaesthesia may be partially due to the opioid shortage in developing countries and partly due to the anticipated reduction of anticipated opioidrelated side effects. Hence, it is also essential to note that certain combinations like multimodal analgesia, regional and opioid-free techniques can also result in detrimental rather than beneficial effects. It is necessary to choose clinically validated regimens specific to certain procedures and patient populations.

Opioid-free and opioid sparing strategies may have the advantage of limiting the side effects of opioids, but they are not devoid of side effects of their own. To better understand these effects, it's essential to conduct good quality research on a larger population with different and new drug combinations to determine the factors that improve overall outcomes and minimize potential adverse effects. Although, the choice of anaesthesia among variety of patients and surgeries depends more on subjective than objective reasons. However, by compiling evidence from diverse clinical practices, a unique group of variables may come up for better decision making. Developing prediction modelling for the choice of anaesthesia may also be possible by including different highly plausible patient related and procedure related characteristics with the aim of minimizing possibility of potential hazards of opioids and non-opioid interventions.

\section{Conflict of interests}

None declared by the authors.

\section{Authors' contribution}

All authors took equal part in the literature search and the manuscript preparation. All authors approve the manuscript.

\section{References}

1. Forget $P$. Opioid-free anaesthesia. Why and how? A contextual analysis. Anaesth Crit Care Pain Med. 2019;38(2):169-172. [PubMed] DOI: 10.1016/j.accpm.2018.05.002

2. Brown EN, Pavone KJ, Naranjo M. Multimodal general anesthesia: theory and practice. Anesth Analg. 2018;127(5):1246-1258. [PubMed] [PubMed] DOI: 10.1213/ANE.0000000000003668
3. Shanthanna H, Ladha K S, Kehlet H, Joshi G P. Perioperative opioid administration. a critical review of opioid-free versus opioid-sparing approaches. Anesthesiology. 2021;134:645-59. [PubMed] DOI: 10.1097/ALN.0000000000003572

4. Ferry N, Dhanjal S. Opioid Anesthesia. In: StatPearls. Treasure Island (FL): StatPearls Publishing; 2021.

5. Preuss CV, Kalava A, King KC. Prescription of Controlled Substances: Benefits and Risks. In: StatPearls. Treasure Island (FL): StatPearls Publishing; 2021.

6. Kharasch ED, Brunt LM. Perioperative opioids and public health. Anesthesiology. 2016;124(4):960-965. [PubMed] DOI: 10.1097/ALN.0000000000001012

7. de Boer HD, Detriche O, Forget P. Opioid-related side effects: Postoperative ileus, urinary retention, nausea and vomiting, and shivering. A review of the literature. Best Pract Res Clin Anaesthesiol. 2017;31(4):499-504. [PubMed] DOl: 10.1016/j.bpa.2017.07.002

8. Toleska M, Dimitrovski A. Is opioid-free general anesthesia more superior for postoperative pain versus opioid general anesthesia in laparoscopic cholecystectomy? Pril (Makedon Akad Nauk Umet Odd Med Nauki). 2019;40(2):81-87. [PubMed] DOI: 10.2478/prilozi-2019-0018

9. Thompson-Brazill KA. Pain control in the cardiothoracic surgery patient. Crit Care Nurs Clin North Am. 2019;31(3):389-405. [PubMed] DOI: 10.1016/j.cnc.2019.05.007

10. Mansour MA, Mahmoud AA, Geddawy M. Nonopioid versus opioid based general anesthesia technique for bariatric surgery: A randomized double-blind study. Saudi J Anaesth. 2013;7:387-391. [PubMed] DOI: 10.4103/1658-354X.121045

11. Dowell D, Haegerich TM, Chou R. CDC Guideline for prescribing opioids for chronic pain--United States. JAMA. 2016;315(15):1624-1645. [PubMed] DOI: 10.1001/jama.2016.1464

12. Kharasch ED, Clark JD. Opioid-free anesthesia: time to regain our balance. Anesthesiology. 2021;134(4):509-514. [PubMed] DOI: 10.1097/ALN.0000000000003705

13. Tempe DK, Sawhney C. Opioid-free anesthesia for thoracic surgery: a step forward. J Cardiothorac Vasc Anesth. 2020;34(11):3041-3043. [PubMed] DOl: 10.1053/j.jvca.2020.07.022

14. Schwenk ES, Mariano ER. Designing the ideal perioperative pain management plan starts with multimodal analgesia. Korean J Anesthesiol. 2018;71(5):345-352. [PubMed] DOI: 10.4097/kja.d.18.00217

15. Medina S. Opioid free anesthesia. Doc Nurs Prac Projects. 2020.129. [FreeFullText]

16. Elkassabany NM, Mariano ER. Opioid-free anaesthesia - what would Inigo Montoya say? Anaesthesia. 2019;74(5):560-563. [PubMed] DOI: 10.1111/anae.14611

17. Thota RS, Ramkiran S, Garg R, Goswami J, Baxi V, Thomas M. Opioid free onco-anesthesia: Is it time to convict opioids? A systematic review of literature. J Anaesthesiol Clin Pharmacol. 2019;35(4):441-452. [PubMed] DOI: 10.4103/joacp.JOACP_128_19

18. Pavone KJ, Jablonski J, Cacchione PZ, Polomano RC, Compton $\mathrm{P}$. Evaluating pain, opioids, and delirium in critically ill 
older adults. Clin Nurs Res. 2021:30(4):455-463. [PubMed] DOI: $10.1177 / 1054773820973123$

19. Rizk E, Haas EM, Swan JT. Opioid-sparing effect of liposomal bupivacaine and intravenous acetaminophen in colorectal surgery. J Surg Res. 2021;259:230-41. [PubMed] DOI: 10.1016/j.jss.2020.09.002

20. Frauenknecht J, Kirkham KR, Jacot-Guillarmod A, Albrecht E. Analgesic impact of intra-operative opioids vs. opioid-free anaesthesia: a systematic review and meta-analysis. Anaesthesia. 2019;74(5):651-662. [PubMed] DOI: 10.1111/anae. 14582

21. Chia PA, Cannesson M, Bui CCM. Opioid free anesthesia: feasible? Curr Opin Anaesthesiol. 2020;33(4):512-517. [PubMed] DOI: 10.1097/ACO.0000000000000878
22. Beloeil H, Garot M, Lebuffe G, Gerbaud A, Bila J, Cuvillon P, et al. Balanced opioid-free anesthesia with dexmedetomidine versus balanced anesthesia with remifentanil for major or intermediate noncardiac surgery. randomized controlled trial. Anesthesiology. 2021;134:541-551. [PubMed] DOI: 10.1097/ALN.0000000000003725

23. Ali ME, Ali IM. Defining an opioid sparing treatment pathway for chronic abdominal pain of somatic and visceral origin: a case series. J Pain Palliat Care Pharmacother. 2021;35(1):43-47. [PubMed] \ DOl: 10.1080/15360288.2020.1828527

24. Pandharipande PP, Pun BT, Herr DL, Maze M, Girard TD, Miller RR, et al. Effect of sedation with dexmedetomidine vs lorazepam on acute brain dysfunction in mechanically ventilated patients: the MENDS randomized controlled trial. JAMA. 2007;298(22):2644-2653. [PubMed] DOI: 10.1001/jama.298.22.2644 